特別 講 演

\title{
Inflammation, Immunity and Remodeling in Chronic Rhinosinusitis
}

\author{
Robert P. Schleimer \\ The Division of Allergy and Immunology, Department of Medicine, \\ Northwestern University Feinberg School of Medicine, Chicago
}

\begin{abstract}
We have been studying the mechanisms of pathogenesis in chronic rhinosinusitis (CRS) in a collaboration of ENT surgeons, allergists and bench scientists. We distinguish between patients with non polypoid CRS (CRSsNP) and those with polypoid CRS (CRSwNP). We collect samples from the inferior turbinate and the uncinate process as well as nasal polyp tissue, from control subjects and subjects with CRSsNP and CRSwNP. We have found marked inflammatory responses in the nasal polyp (NP) tissue from CRSwNP patients, characterized by elevations in B cells, plasma cells, $\mathrm{T}$ cells, dendritic cells, neutrophils, eosinophils and mast cells. We have detected evidence for a defect in innate immunity in CRS patients, as measured by reduced expression of several host defense molecules, including members of the S100 and PLUNC family of genes. This defect may help to explain the frequent colonization of the upper airways from which these patients suffer. Colonization with staph aureus and other microbes also probably explains an
\end{abstract}

enhancement of adaptive immune cells and the presence of local immunoglobulin expression that we observe in CRSwNP. We have recently addressed the possible explanation for why NP grow in the ethmoid region but not the inferior turbinate and have new evidence suggesting that NP are composed of substantial quantities of cross linked fibrin and that this results from low levels of t-PA in CRS patients. T-PA is important in degrading fibrin by activating plasminogen, and the reduced levels of t-PA may result from enhancement of Th2 cytokines in CRSwNP. We also find elevated levels of factor XIIIA, which is important in forming crosslinked fibrin. Changes in t-PA and factor XIIIA may synergize to form polyp tissue. The strong adaptive immune response that we observe in CRS extends to include the presence of autoantibodies in CRSwNP, and we have evidence to suggest that the TNF family cytokine BAFF, chemokines such as BLC and SDFla and the 7TM receptor EBI2 all play a role in promoting the local antibody response. 